\title{
Poloxamer 188 as a Supplement to Barium Cross-Linked Ultra-High Viscosity Alginate for Immunoisolation of Transplanted Islet Cells
}

\author{
Mettler $\mathrm{E}^{1}$, Zimmermann U, Hansen $\mathrm{T}^{2}$, Ehrhart $\mathrm{F}^{3}$, Zimmermann $\mathrm{H}^{3}$ and Weber $\mathrm{MM}^{1}$
}

${ }^{1}$ Department of Endocrinology and Metabolism, University Medical Center Mainz, Germany

${ }^{2}$ Institute of Pathology, University Medical Center Mainz, Germany

${ }^{3}$ Fraunhofer Institute for Biomedical Technology, Ensheimer Str. 48, 66386, St. Ingbert, Germany

\begin{abstract}
Transplantation of Langerhans islets is a potential cure for diabetes mellitus. The main problem for routine clinical use remains the prevention of rejection without drastic side effects. Immuno-isolation is an experimental strategy to prevent graft rejection by separating the transplanted cells from the host immune system using a barrier device. The aim of the current study was to improve the physical features of encapsulated islets in a barium cross-linked ultra-high viscosity alginate by adding Poloxamer 188 (P188). Empty alginate capsules, and especially encapsulated islets, could be easily generated using UHV-P188 alginate because of its anti-foaming properties. Diabetic mice were used for evaluation of biocompatibility and graft function. Biocompatibility testing with empty capsules showed no inflammatory reaction or fibrotic overgrowth. The capsules remained intact in the intraperitoneal and intramuscular implant sites over a period of 4 weeks. Transplantation of encapsulated islets, however, led to a strong systemic inflammatory response with fibrotic overgrowth of the islet-containing capsules but no graft failure. This finding likely reflected the complementactivating property of P188. Our results clearly showed that the complex interaction of additives, xenogeneic tissue, and alginate with the host immune system could not be predicted by the behavior of the individual components. Furthermore, the mouse model described herein was an excellent tool to evaluate the physico-chemical properties and the in vivo biocompatibility and functionality of various additives. Our results will improve the biomaterials used for alginate microbeads in a clinical setting in the future.
\end{abstract}

Keywords: Microencapsulation; Islets of langerhans; Diabetes mellitus; Xenogeneic transplantation; Alginate; Islet transplantation; Poloxamer 188

Abbreviations: IEq: Islet Equivalents; P188: Poloxamer 188; GSIS: Glucose-Stimulated Insulin Secretion; Stz: Streptozotocin; M: D-Mannuronic Acid; G: L-Guluronic Acid; UHV: Ultra High Viscosity Alginates

\section{Introduction}

We have been investigating rat pancreatic xenografts as a potential source of functional islet tissue for transplantation using a mouse model. The goal of this work was to develop a therapeutic strategy to overcome the shortage of donor organs for the treatment of diabetes mellitus. To prevent strong humoral and cellular immune responses to the xenografts, we tested alginate microcapsules as an immunoisolation device. Immunoisolation technology is based on the principle that the artificial membrane protects foreign cells from the host immune system while simultaneously allowing unopposed transfer of nutrients, oxygen, and therapeutic factors [1].

According to our previously published results [2], the use of ultra-high viscosity (UHV) alginate for microencapsulation provided long-term functional rat and human islets after intraperitoneal xenotransplantation into immunocompetent diabetic mice, without the need for immunosuppression.

The composition and sequential structure of the alginate are of great importance when used as an encapsulating material. Alginate is usually isolated from marine brown algae, and its composition varies widely depending on the subspecies and growth location [3]. Depending on the nature of the cross-linking cation, the length of the polymeric chains, the mannuronic acid:guluronic acid (M:G) ratio, and the percentage of block structures, hydrogels of varying mechanical strength, elasticity, and swelling characteristics can be produced [4]. Alginates with a high content of guluronic acid block polymers produce more solid and less elastic gels with high permeability compared to alginates with a high content of mannuronic acid [5]. Therefore, the M:G ratio, length of polymeric chains, and ratio of homologous to heterologous chains must be carefully tuned to optimize the microcapsules [6].

A lack of standardization and information from manufacturers about the composition of the alginate (e.g., molecular weight, content, and ratio of D-mannuronic acid and L-guluronic acid) is a major problem for encapsulation experiments; this knowledge is of enormous importance for understanding the physico-chemical characteristics and hence biocompatibility of the alginate. $\mathrm{Ba}^{2+}$-gelled capsules comprised of 1:1 mixtures of UHV alginates from the closely related species Lessonia nigrescens and Lessonia trabeculata meet the demands of high stability and flexibility (UHV: 35\% M and 65\% G) [7].

The aims of the current study were 1) to improve the physical features of barium cross-linked UHV alginate by the addition of Poloxamer 188 (P188) as a detergent, and 2) to establish the muscle as a novel transplantation site.

Poloxamer is comprised of ABA-type block polymers that consist of a central, hydrophobic block of polypropylene oxide that is edged by two hydrophilic blocks of polyethylene oxide. Poloxamer 188 (P188),

*Corresponding author: Mettler E, Department of Endocrinology and Metabolism University Medical Center, Mainz, Germany, Tel: +49 6131-17 5909; E-mail: esther. mettler@unimedizin-mainz.de

Received November 16, 2015; Accepted December 07, 2015; Published December 09, 2015

Citation: Mettler E, Zimmermann U, Hansen T, Ehrhart F, Zimmermann H, et al. (2015) Poloxamer 188 as a Supplement to Barium Cross-Linked Ultra-High Viscosity Alginate for Immunoisolation of Transplanted Islet Cells. Metabolomics 5 : 157. doi:10.4172/2153-0769.1000157

Copyright: ( 2015 Mettler E, et al. This is an open-access article distributed under the terms of the Creative Commons Attribution License, which permits unrestricted use, distribution, and reproduction in any medium, provided the original author and source are credited. 
also called Luterol ${ }^{\circ} \mathrm{F} 68$, has an average molecular weight of $\sim 8600$ $\mathrm{Da}$. The polyoxyethylene units represent approximately $81 \%$ of the compound [8]. P188 has diverse applications in various biomedical fields ranging from drug delivery, gene transfer, tissue engineering [9], and vaccine adjuvanting [10] to medical imaging for the management of vascular diseases and disorders, and cancer therapy. We chose P188 as a supplement for the alginate because of its lack of toxicity and its non-thrombogenic properties $[11,12]$.

Many transplantation sites for islets in animal models have been reported (e.g., kidney [13], peritoneum [2], omentum [14], muscle [15], testis [16], and bone marrow [17]); however, in humans, the liver remains the only transplantation site that is used in a clinical setting. Due to the comparatively large size of alginate-encapsulated islets, which significantly increases the risk for portal thrombosis, the liver is not a suitable transplantation site for microencapsulated islet cells. As an alternative site, the muscle is a preferred location because it is highly vascularized and easily accessible if graft removal becomes necessary [18]. Successful auto-transplantation of parathyroid glands [19] and islets [20] into the forearm has been reported. However, for xenotransplantation of encapsulated islets, the requirements for a mechanically stable and highly biocompatible encapsulation material are significantly higher for implantation into muscle compared to subcutaneous tissue or the peritoneum due to problems with mechanical sheer forces. Therefore, we investigated the muscle as a transplantation site for UHV-P188 alginate-encapsulated islets to increase the stability and biocompatibility of the capsules.

\section{Materials and Method}

\section{Islet cell isolation and viability assessment}

Islets of Langerhans were isolated from healthy Wistar rats by a collagenase digestion method. In brief, $10 \mathrm{ml}$ Collagenase solution containing 20 PZ-U NB1, $1 \mathrm{U}$ NP and $1 \mathrm{mg}$ AEBSF (Serva, Germany) were slowly injected into the common bile duct after occlusion of the distal end just proximal to the duodenum. The distended pancreas was excised and the digestion was performed in a waterbath at $37^{\circ} \mathrm{C}$ for $20 \mathrm{~min}$. The tissues were incubated with UW solution for $30 \mathrm{~min}$. Islet purification was achieved using a three-step, discontinuous density gradient (Biochrom, Germany). Islets were collected from the interface between the 1.077 and $1.038 \mathrm{~g} / \mathrm{ml}$ layer. All animal procedures were approved by our institution's Ethics Committee (Landesuntersuchungsamt Rheinland-Pfalz) and carried out under license (no. G 12-1-068), in accordance with the Ethical Committee for Animal Research at the University Medical Center, Mainz, Germany. After purification, islets were recovered overnight at $37^{\circ} \mathrm{C}$ and $5 \% \mathrm{CO}_{2}$ in TCM 199 (Biochrom) supplemented with 1\% Penicillin /Streptomycin (Invitrogen) and 10\% FCS (Seromed Biochrom KG). Single cells and small cell aggregates were removed from the islet suspension by filtration through a $40 \mu \mathrm{m}$ Nylon Cell Strainer (BD Falcon). The volume of isolated islet was expressed as the number of islet equivalents (IEq), defined as an islet $150 \mu \mathrm{m}$ in diameter. The Ricordi algorithm was used to convert islet number into IEq [21].

Viability of islets was assessed using fluorescein diacetate (FDA) (Sigma-Aldrich, St. Louis, MO, USA) and PI (Sigma-Aldrich) staining. Briefly, islets in the inserts were transferred in PBS. $8 \mu \mathrm{FDA}(5 \mathrm{mg} / \mathrm{ml}$ in acetone) and $50 \mu \mathrm{PI}(0.5 \mathrm{mg} / \mathrm{ml}$ in PBS) stock solutions were added to the sample. After $30 \mathrm{~s}$ of adding the stain islets were placed on the fluorescent microscope, visualized and photographed using the filter FITC for fluorescein $(\mathrm{em} \sim 530 \mathrm{~nm})$ and texas red $(\mathrm{em}>615 \mathrm{~nm})$. Dead cells were stained red and viable cells were stained green. For computer- based viability determination of multicellular systems we used a method based on colour intensity of the live-dead stain as previously proposed and used for pancreatic islets [17]. The software used was ImageJ 1.34 $\mathrm{s}$ (NIH, USA). The pictures were split into three colour channels: red, green and blue. The islets were manually marked in both, the green and the red channel, and the mean intensity (of each channel) of the islet area was automatically given. Viability was calculated as followed: Viability [\%] =living cells/ (living+dead cells) ${ }^{*} 100 \%$

\section{Alginat droplet generation and islet encapsulation}

Production of UHV-alginates was performed as described previously [22]. UHV alginates of clinical grade were used for all islet encapsulations (viscosity of a $0.1 \% \mathrm{wt} / \mathrm{vol}$ solution in distilled water was $20-30 \mathrm{mPa} \cdot \mathrm{s}$ ). $0.017 \%$ Poloxamer (BASF) was added to generate UHV-P188 alginate. For microcapsule formation, the alginate was dissolved in sterile, endotoxin-free $0.9 \% \mathrm{NaCl}$ solution at a concentration of $0.65 \%$ (wt/vol). The islets were mixed carefully with the alginate in a concentration of $8000 \mathrm{IEq} / \mathrm{ml}$ alginate solution. Alginate droplets were produced by an air-jet droplet generator and cross-linked using the crystal gun method with external and internal $\mathrm{Ba}^{2+}$ as described previously by Zimmermann et al. [18]. Briefly, air pressure was used to drive the dry, sterile $\mathrm{BaCl}_{2}$ crystals into the alginate droplets before they reached the cross-linking solution, leading to internal gelling. External gelling occurred when the droplets came into contact with a $20 \mathrm{mM} \mathrm{BaCl}_{2}$ solution. The osmolality of the cross-linking solution was adjusted to $290 \mathrm{mOsm}$ using appropriate amounts of $\mathrm{NaCl}$. After 20 minutes, the alginate/islet capsules were washed three times with $0.9 \%$ $\mathrm{NaCl}$ solution.

\section{In vivo biocompatibility of microcapsules}

To assess the biocompatibility of empty alginate microcapsules, the droplets were implanted by intraperitoneal or intramuscular injection into healthy BALB/c mice or NOD mice. Microcapsules were counted, measured by size and by volume before transplanted under sterile conditions. Control animals received the same volume of $0.9 \% \mathrm{NaCl}$ solution. Grafts were retrieved 4 weeks after transplantation.

Intraperitoneal grafts were explanted by repeated flushes with warm $0.9 \% \mathrm{NaCl}$ solution containing $20 \mathrm{mM} \mathrm{BaCl}_{2}$ Intramuscular grafts were surgically removed. The microcapsules were removed and the percentage of recovered capsules (defined as the ratio to the volume of capsules before transplantation to that after removal) and the degree of capsular overgrowth, defined as the number of capsules presenting adherent cells on their surface per 100 capsules, were determined. Images were made with a digital camera and stored as jpg files.

\section{In vivo test of graft function}

Transplantation experiments were carried out with healthy, 5-6-week-old BALB/c mice or spontaneously diabetic NOD mice. $\mathrm{BALB} / \mathrm{c}$ mice were rendered diabetic with intraperitoneal injections of streptozotocin (stz $250 \mathrm{mg} / \mathrm{kg}$ body wt; Sigma, St. Louis, MO, USA) freshly dissolved in citrate buffer 2 weeks before islet transplantation. Only animals exhibiting blood glucose higher than $350 \mathrm{mg} / \mathrm{dl}$ in two consecutive measurements were considered diabetic and were used as recipients. German guidelines for the care and use of experimental animals were strictly followed throughout the study. Each mouse received 2000 microencapsulated IEq. Transplants were made in a class 100 biological safety cabinet under sterile conditions. Islets were injected into the peritoneal cavity with a 20-gauge needle (Vasofix; Braun, Melsungen, Germany). Non fasting blood glucose level of transplanted mice was monitored every 3 days, later weekly. FreeStyle 
Citation: Mettler E, Zimmermann U, Hansen T, Ehrhart F, Zimmermann H, et al. (2015) Poloxamer 188 as a Supplement to Barium Cross-Linked UltraHigh Viscosity Alginate for Immunoisolation of Transplanted Islet Cells. Metabolomics 5: 157. doi:10.4172/2153-0769.1000157

Page 3 of 5

mini (Abbott, Germany) was used for blood glucose determination. Grafts were removed after graft failure or poor general condition of the animals. Stz control mice received every 3-4 days subcutaneous administration of insulin (Novo Nordisk Levemir, 3-4 international units/injektion).

Explanted microcapsules were counted, measured by size and by volume as well as evaluated for fibrotic overgrowth using an IX70 microscope (Olympus). Explanted encapsulated islets were fixed in paraffin blocks.

\section{Assessment of insulin secretion}

The static incubation assay was used to assess the insulin secretion response of islets after a glucose challenge, as previously described [23]. $50 \mathrm{IEq}$ were transferred into a culture insert (membrane pore diameter $12 \mu \mathrm{m}$; Millicell PCF, Millipore, France) and put into a well of a 24-well culture-plate (Falcon Multiwell; Becton, Dickinson). Six wells were prepared for each experimental group. Basal insulin secretion was measured after $60 \mathrm{~min}$ of incubation at $37^{\circ} \mathrm{C}$ in Medium 199 $+10 \%$ FCS. The inserts with islets were transferred into hyperglycemic culture medium (Medium199 + 10\% FCS + $200 \mathrm{mg} / \mathrm{dl}$ D-glucose) for additional 60 minutes. The supernatant was frozen at $-21^{\circ}$ until determining the insulin concentration using sandwich ELISA. ELISAplates (high binding; Greiner) were coated with $1 \mu \mathrm{g} / \mathrm{ml}$ mouse $\mathrm{mAb}$ to insulin (abcam, Cambridgeshire, UK) diluted in $0.1 \mathrm{M}$ bicarbonate buffer, $\mathrm{pH}$ 9.6. Plates were incubated overnight at $4^{\circ} \mathrm{C}$. After incubation, plates were washed four times with PBS containing $0.05 \%$ Tween 20 and blocked for $2 \mathrm{~h}$ at $37^{\circ} \mathrm{C}$ by adding $50 \mu \mathrm{l}$ of blocking solution (Candor, Germany) to each well. Thawed supernatants and insulin standards (Sigma, St. Louis, MO, USA) were added at a volume of $35 \mu \mathrm{l} /$ well (1:3,5 in PBS). Plates were incubated overnight at $4^{\circ} \mathrm{C}$ then washed. 0.15 $\mu \mathrm{g} / \mathrm{ml}$ of secondary HRP conjugated mouse $\mathrm{mAb}$ to insulin (abcam, Cambridgeshire, UK) diluted in low cross binding buffer (Candor, Germany) was added at $25 \mu \mathrm{l} /$ well. Plates were incubated for $2 \mathrm{~h}$ at room temperature. After washing, plates were developed by adding 25 $\mu \mathrm{l} /$ well of TMB (Sigma, St. Louis, MO, USA). The reaction was stopped by adding $25 \mu \mathrm{l} /$ well of $1 \mathrm{~N} \mathrm{H}_{2} \mathrm{SO}_{4}$. The extinction was measured in a ELX800G (Bio-Tek Instruments, Winooski, USA) equipped with KC4 software using a reference wavelength of $630 \mathrm{~nm}$ and a test wavelength of $450 \mathrm{~nm}$.

\section{Immunhistochemistry}

In brief, serial sections $(5 \mu \mathrm{m})$ were cut from $5 \%$ formaldehydefixed paraffin-embedded pancreas or retrieved graft tissue. Serial sections $(5 \mu \mathrm{m})$ were deparaffinized, rehydrated, and subjected to antigen unmasking with citrate buffer ( $\mathrm{pH}$ 6.0).

Sections were treated with $1 \%$ bovine serum albumin (BSA) in phosphate-buffered saline (PBS) for $60 \mathrm{~min}$ to block nonspecific antibody binding. The primary antibody (chicken polyclonal antiinsulin antibody (1:100; abcam, Cambridgeshire, UK) was diluted with PBS containing 1\% BSA and applied to the sections. Rabbit anti chicken HRP (abcam, Cambridgeshire, UK) was used as secondary antibody, diluted 1:200 in PBS and incubated for $60 \mathrm{~min}$. Insulin was identified with 3,3区diaminobenzidine tetrachloride (H-2200, DAB, Daco, Germany) and sections were counterstained with Mayer hematoxylin. Digital images of the stained slices were obtained using Olympus IX70 microscope.

\section{Statistics}

All experiments were repeated a minimum of three times. Data are reported as means \pm S.D. All statistical analyses were performed using SPSS 12.0 (SPSS Inc., Chicago, IL, USA). Significant differences were identified using Levene's Test for equality of variances and independent samples t-test. In all tests, the significance level was set at 5\% $(\mathrm{P}<0.05)$.

\section{Results}

\section{In vitro evaluation of UHV-P188 alginate-microencapsulated islets}

Before transplantation of the encapsulated islets, their insulin secretory capacity was determined after 1 day of culture. As shown in Figure 1, encapsulated rat islets responded well to a high-glucose stimulus. There was no significant difference in insulin secretion and release into the medium between the non-encapsulated islets and the microencapsulated islets after glucose challenge.

\section{Biocompatibility of empty alginate capsules}

The biocompatibility of pure UHV-P188 alginate droplets was tested by intramuscular (im) and intraperitoneal (ip) transplantation into healthy $\mathrm{BALB} / \mathrm{c}$ and diabetic NOD mice. Five weeks after transplantation, the retrieved empty alginate droplets did not exhibit any cellular overgrowth on their surfaces (Figure 2).

\section{Graft function in vivo}

Graft function was tested in diabetic mice. Specifically, diabetes was induced chemically (streptozotocin, stz) in BALB/c mice; diabetes developed spontaneously in NOD mice. Non-fasting blood glucose levels in the transplanted stz diabetic $\mathrm{BALB} / \mathrm{c}$ mice and spontaneously diabetic NOD mice were monitored. The mice were transplanted ip or im with microencapsulated islets. Normoglycemic blood glucose levels were generally reached within $24 \mathrm{~h}$ of transplantation and lasted up to 15 days in the NOD mice and $>70$ days in the BALB/c mice (Figure 3 $a$ and $3 b)$.

Despite normoglycemia, the general condition of the transplanted $\mathrm{BALB} / \mathrm{c}$ mice progressively deteriorated, as shown by weight loss and dramatic leukocytosis, so that the animals had to be sacrificed on day 76. Approximately $25 \%$ of the explanted encapsulated islets showed mild to moderate cellular overgrowth (Figure $3 \mathrm{c}$ and $3 \mathrm{~d}$ ). When the transplantation experiment was repeated, the same deterioration was observed and the experiment had to be stopped, despite functional islet grafts.

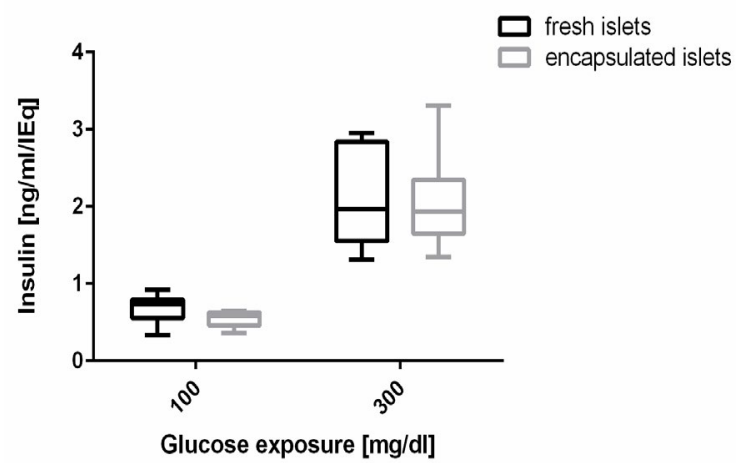

Figure1: In vitro insulin-release from fresh and microencapsulated islets A static incubation assay was performed with fresh isolated islets and islets microencapsulated in UHV-P188 alginate. Insulin secretion was measured in supernatants using Insulin ELISA. Results are representative of three separate cell isolation and encapsulation experiments. * marks an outlier. 
Citation: Mettler E, Zimmermann U, Hansen T, Ehrhart F, Zimmermann H, et al. (2015) Poloxamer 188 as a Supplement to Barium Cross-Linked UltraHigh Viscosity Alginate for Immunoisolation of Transplanted Islet Cells. Metabolomics 5: 157. doi:10.4172/2153-0769.1000157

\section{Recovery and examination of the explanted grafts}

All microencapsulated islets retrieved from NOD mice 25 days after transplantation showed massive cellular attachment on their surfaces (Figure $3 \mathrm{e}$ and $3 \mathrm{f}$ ). There was no transplantation site inflammatory reaction observed in either set of mice. Immunohistochemical staining of pancreatic tissues from stz diabetic mice confirmed the loss of beta cell mass (Figure $4 \mathrm{a}$ and $4 \mathrm{~b}$ ). Microencapsulated islet grafts retrieved 76 days post-transplantation were positive for insulin by immunohistochemistry, which verified the presence of viable beta cells (Figure 4c). The loss of beta cell mass within the capsule is due to the massive fibrotic cellular attachment.

\section{Discussion}

The success of alginate microbeads as an immunoisolation device depends largely on the bio-physico-chemical properties of the microbeads [24]. In the present study, we investigated P188 as an alginate supplement to improve alginate capsule biocompatibility and graft function.

We were able to demonstrate stable physico-chemical properties of the alginate capsules in vitro. The alginate capsules had high viability and retained regulated insulin secretory capacity and very good biocompatibility in vivo without any fibroblast overgrowth reaction after the transplantation of empty capsules. However, we found that the addition of $\mathrm{P} 188$ resulted in an unexpected immunogenic reaction when encapsulated islets were transplanted intraperitoneally or intramuscularly. These results suggested that P188 in the presence of xenogeneic tissue behaved as an immunogenic adjuvant.

A series of poloxamers has been tested for adjuvant activity using a standard oil-in-water emulsion, with egg albumin as the antigen [20].

Adjuvant activity studies of copolymers showed that antibody titers generally increased with increasing molecular weight of the hydrophobic poly(oxypropylene)amine salts (POP), along with decreasing content of hydrophilic polyoxyethylene (POE). Pure polymers of POP, however, were not as effective as adjuvants [12]. P188 does have a relatively high molecular weight $(\sim 8400 \mathrm{Da})$ and a high hydrophilic POE content of 35\% [11]. Moghimi et al. [9] described the ability of P188 to activate the alternative complement pathway. Based on these results, the use of a higher molecular weight poloxamer as an alginate additive for microencapsulation of islet cells warrants further study. A promising candidate is Poloxamer 407, which has been shown to prevent nonspecific binding of IgG to the solid surface and phagocytosis by neutrophils [25].

The peritoneum is a classic site for the transplantation of microcapsules because a large amount of capsules can be easily injected. However, the peritoneum provides an ideal environment for inflammatory and immunological reactions [26]. Peritoneal mesothelial cells facilitate the induction and promotion of a strong innate immune reaction to antigen. Recently, studies of encapsulated pig islets transplanted into mice reported that the biocompatibility was improved and the survival of encapsulated pig islet transplants was prolonged by the inhibition of macrophage and lymphocyte stimulation [27]. Therefore, we attempted to evaluate a site that would be less immunoreactive for implantation of islet xenograft microcapsules, and that would also avoid the need for immunosuppression. Liver and spleen were excluded directly as graft sites because the transplantation of capsules having a diameter of $\sim 500 \mu$ m greatly increases the risk for thrombosis. Therefore, intramuscular transplantation of encapsulated islets offers an attractive alternative, based on its simplicity and ease of

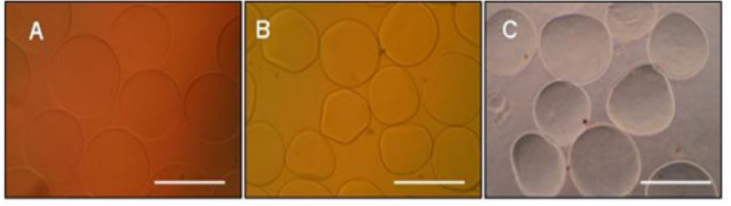

Figure2: Representative images of fresh (A) and explanted (B-C) UHV-P188 alginate capsules without encapsulated islets of Langerhans

Light microscopy of Ba2+ cross-linked UHV-P188 alginate capsules recovered 6 weeks after intraperitoneal implantation (B) or intramuscular implantation (C) into BALB/c and NOD mice. Bar scale $=500 \mu \mathrm{m}$.
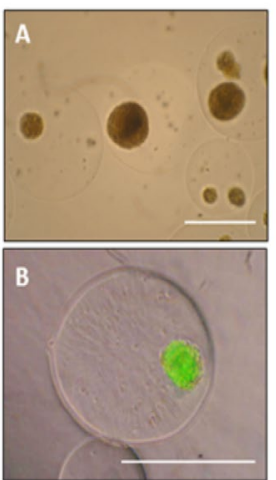
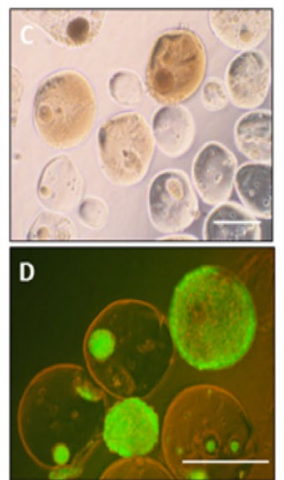

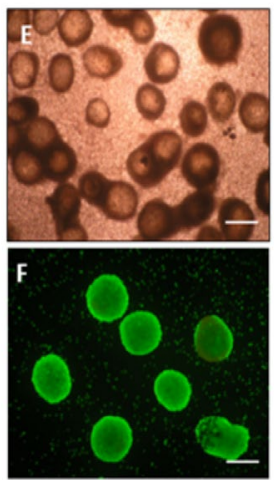

Figure 3: Representative images of fresh and retrieved microencapsulated islets

Light microscopic $(A, C, E)$ and fluorescent microscopic images $(B, D, F)$ of microencapsulated islets. Pictures were taken after encapsulation $(A, B)$, at 76 days after transplantation into BALB/C mice $(C, D)$, or at 20 days after transplantation into NOD mice $(E, F)$. Viability staining with fluorescein diacetate (FDA; green=viable) and propidium iodide ( $\mathrm{Pl}$; red=dead) of encapsulated islets. Bar scale- $500 \mu \mathrm{m}$.
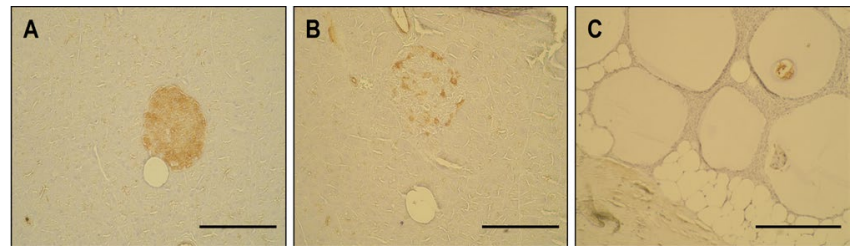

Figure 4: Representative images of immunohistochemical staining of pancreatic islets for insulin expression

(A) Pancreatic sections from a healthy BALB/c mouse. (B) Pancreatic section from an stz diabetic BALB/c mouse with a functioning graft on day 76 posttransplantation. (C) Section from an im-transplanted microencapsulated islet graft on day 76 post-transplantation.

Bar scale $(A, B) 200 \mu \mathrm{m}$, (C) $500 \mu \mathrm{m}$.

access for noninvasive graft imaging and cell explantation.

Alginate microbeads have been investigated clinically for a number of therapeutic interventions, including drug delivery, cell delivery, and cell encapsulation [28]. Studies have shown promising results in these applications; however, clinical success requires the ability to control and predict solute transport in the alginate matrix, as well as possible interactions between the delivered drug/tissue, the alginate, and the host system. Our results clearly showed that the biocompatibility of such a complex system cannot be predicted by the behavior of its individual components; the complex interactions, especially in the setting of transplanted, immunoseparated xenogeneic tissue that has been encapsulated, should be tested in vivo. The mouse 
Citation: Mettler E, Zimmermann U, Hansen T, Ehrhart F, Zimmermann H, et al. (2015) Poloxamer 188 as a Supplement to Barium Cross-Linked UltraHigh Viscosity Alginate for Immunoisolation of Transplanted Islet Cells. Metabolomics 5: 157. doi:10.4172/2153-0769.1000157

Page 5 of 5

model of xenotransplantation of islets encapsulated with our highly defined UHV alginate was excellent for the in vivo study of the physicochemical properties of the alginate microbeads. The model also was ideal for testing the in vivo biocompatibility and functionality of the islet cell graft.

\section{Acknowledgement}

Research funding was provided by grants from the Bundesministerium für Bildung und Forschung (BMBF), Germany given to MMW (0315818 C) and HZ (0315818 B).

\section{References}

1. Krishnamurthy NV, Gimi B (2011) Encapsulated cell grafts to treat cellular deficiencies and dysfunction. Crit Rev Biomed Eng 39: 473-491.

2. Schneider S, Feilen PJ, Brunnenmeier F, Minnemann T, Zimmermann $\mathrm{H}$, et al. (2005) Long-Term Graft Function of Adult Rat and Human Islets Encapsulated in Novel Alginate-Based Microcapsules After Transplantation in Immunocompetent Diabetic Mice. Diabetes 54: 687-693.

3. De Vos P, Faas MM, Strand B, Calafiore R (2006) Alginate-based microcapsules for immunoisolation of pancreatic islets. Biomaterials 27: 5603-5617.

4. Martinsen A, Skjåk-Braek G, Smidsrød O (1989) Alginate as immobilization material: I. Correlation between chemical and physical properties of alginate gel beads. Biotechnol Bioeng 33: 79-89.

5. De Vos P, van Hoogmoed CG, van Zanten J, Netter S, Strubbe JH, et al. (2003) Long-term biocompatibility, chemistry, and function of microencapsulated pancreatic islets. Biomaterials 24: 305-312.

6. Zimmermann H, Wählisch F, Baier C, Westhoff M, Reuss R, et al. (2007) Physical and biological properties of barium cross-linked alginate membranes. Biomaterials 28: 1327-1345.

7. Zimmermann H, Zimmermann D, Reuss R, Feilen PJ, Manz B, et al. (2005) Towards a medically approved technology for alginate-based microcapsules allowing long-term immunoisolated transplantation. J Mater Sci Mater Med 16: 491-501.

8. http://worldaccount.basf.com/wa/NAFTA/Catalog/Pharma/info/BASF/exact/ lutrol $f 68$.

9. Moghimi SM, Hunter AC, Dadswell CM, Savay S, Alving CR, et al. (2004) Causative factors behind poloxamer 188 (Pluronic F68, FlocorTM)-induced complement activation in human sera: A protective role against poloxamermediated complement activation by elevated serum lipoprotein levels. Biochimica et Biophysica Acta1689: 103-113.

10. Fox CB, Anderson RC, Dutill TS, Goto Y, Reed SG, et al. (2008) Monitoring the effects of component structure and source on formulation stability and adjuvan activity of oil-in-water emulsions. Colloids Surf B Biointerfaces 65: 98-105.

11. Singh-Joy SD, McLain VC (2008) Safety assessment of poloxamers 101, 105 $108,122,123,124,181,182,183,184,185,188,212,215,217,231,234$ $235,237,238,282,284,288,331,333,334,335,338,401,402,403$, and 407 poloxamer 105 benzoate, and poloxamer 182 dibenzoate as used in cosmetics. Int J Toxicol 27 Suppl 2: 93-128.
12. Schmolka I (1977) A review of block polymer surfactants. J Am Oil Chem Soc 54: $110-116$

13. Sakata N, Chan NK, Chrisler J, Obenaus A, Hathout E (2010) Bone Marrow Cell Co-Transplantation with Islets Improves their Vascularization and Function. Transplantation 89: 686-693.

14. McQuilling JP, Arenas-Herrera J, Childers C, Pareta RA, Khanna O, et al (2011) New Alginate Microcapsule System for Angiogenic Protein Delivery and Immunoisolation of Islets for Transplantation in the Rat Omentum Pouch. Transplant Proc 43: 3262-3264.

15. Christoffersson G, Carlsson P-O, Phillipson M (2011) Intramuscular islet transplantation promotes restored islet vascularity. Islets 3: 69-71.

16. Gores PF, Hayes DH, Copeland MJ, Korbutt GS, Halberstadt C, et al. (2003) Long-term survival of intratesticular porcine islets in nonimmunosuppressed beagles. Transplantation 75: 613-618

17. Kover K, Tong PY, Pacicca D, Clements M, Bodker AM, et al. (2011) Bone marrow cavity: a supportive environment for islet engraftment. Islets 3: 93-101.

18. Sakata N, Sumi S, Yoshimatsu G, Goto M, Egawa S, et al. (2012) Encapsulated islets transplantation: Past, present and future. World J Gastrointest Pathophysiol 3: 19-26.

19. Tominaga Y, Uchida K, Haba T, Katayama A, Sato T, et al. (2001) More than 1,000 cases of total parathyroidectomy with forearm autograft for renal hyperparathyroidism. Am J Kidney Dis 38: S168-S171.

20. Rafael E, Tibell A, Rydén M, Lundgren T, Sävendahl L, et al. (2008) Intramuscular autotransplantation of pancreatic islets in a 7-year-old child: a 2-year follow-up. Am J Transplant 8: 458-462.

21. Ricordi C, Gray DW, Hering BJ, Kaufman DB, Warnock GL, et al. (1990) Islet isolation assessment in man and large animals. Acta Diabetol Lat 27: 185-195.

22. Storz H, Müller KJ, Ehrhart F, Gómez I, Shirley SG, et al. (2009) Physicochemical features of ultra-high viscosity alginates. Carbohydr Res 344: 985-995.

23. Mettler E, Trenkler A, Feilen PJ, Wiegand F, Fottner C, et al. (2013) Magnetic separation of encapsulated islet cells labeled with superparamagnetic iron oxide nano particles. Xenotransplantation. 20:219-26.

24. Zimmermann H, Ehrhart F, Zimmermann D, Müller K, Katsen-Globa A, et al. (2007) Hydrogel-based encapsulation of biological, functional tissue: fundamentals, technologies and applications. Appl Phys A 89: 909-922.

25. Kustiawan I, Derksen NIL, Rispens T (2013) Preventing adsorption of immunoglobulin $\mathrm{G}$ to solid surfaces using poloxamer 407 eliminates artifactual stimulation of neutrophils. J Immunol Methods. 392:49-56.

26. Hall JC, Heel KA, Papadimitriou JM, Platell C (1998) The pathobiology of peritonitis. Gastroenterology 114: 185-196.

27. Cui H, Tucker-Burden C, Cauffiel SMD, Barry AK, Iwakoshi NN, et al. (2009) Long-term metabolic control of autoimmune diabetes in spontaneously diabetic nonobese diabetic mice by nonvascularized microencapsulated adult porcine islets. Transplantation 88: 160-169.

28. Moya ML, Morley M, Khanna O, Opara EC, Brey EM (2012) Stability of alginate microbead properties in vitro. J Mater Sci Mater Med 23: 903-912. 